\title{
RANKINGS GENERATED BY SPHERICAL DISCRIMINANT ANALYSIS
}

\author{
Hidehiko Kamiya* and Akimichi Takemura**
}

\begin{abstract}
We consider the same problem as in Kamiya and Takemura (1997), but for discriminant analysis on $(n-1)$-dimensional unit sphere $S^{n-1}$. That is, we regard pairwise discriminant analysis of $m$ populations on $S^{n-1}$ as a process to generate rankings among the populations, and give a formula for the number of generated rankings.
\end{abstract}

Key Words and Phrases: ranking, discriminant analysis, arrangement of hyperplanes, Poincaré polynomial.

\section{Introduction}

In a recent paper (Kamiya and Takemura (1997), abbreviated as KT throughout the paper), we investigated the properties of rankings associated with linear discriminant analysis among $m$ populations in $R^{n}$. In that paper, we regarded multiple linear discriminant analysis as a process to generate rankings among the populations, and gave formulae for the number of generated rankings, and moreover, basic characterizations of non-generated rankings.

In the present paper, we consider the same problem for discriminant analysis on $(n-1)$-dimensional unit sphere $S^{n-1}=\left\{x \in R^{n}:\|x\|=1\right\}$. Suppose we are given $m$ populations on $S^{n-1}$. For each pair of the populations, we take an $(n-2)$-dimensional discriminant boundary. Then $S^{n-1}$ is divided into regions by $m(m-1) / 2$ such boundaries, and each region is indexed by a ranking among the $m$ populations according to the proximity to them. In this way, we can regard pairwise discriminant analysis on $S^{n-1}$ as a process to generate rankings among the populations.

Let $m$ populations be the von Mises-Fisher distributions $M_{n}\left(\boldsymbol{\mu}_{i}, \kappa\right), i=1, \cdots$, $m$, with common concentration parameter $\kappa>0$ :

$$
\boldsymbol{x}=\left(x_{1}, x_{2}, \cdots, x_{n}\right)^{\prime} \sim c_{n}(\kappa) e^{\kappa \mu i x} d S_{n-1},
$$

where $c_{n}(\kappa)$ is the normalizing constant, $\boldsymbol{\mu}_{i}=\left(\mu_{1 i}, \mu_{2 i}, \cdots, \mu_{n i}\right)^{\prime} \in S^{n-1}, i=1, \cdots, m$, and $d S_{n-1}$ is the volume element on $S^{n-1}$ (Mardia, Kent, and Bibby (1979)).

Assuming equal prior probability for the $m$ populations, we follow the following pairwise allocation rule: Assign a subject $x=\left(x_{1}, x_{2}, \cdots, x_{n}\right)^{\prime} \in S^{n-1}$ to population $i$ in preference to population $j$ iff

$$
c_{n}(\kappa) e^{\kappa \mu_{i} x}>c_{n}(\kappa) e^{\kappa \mu^{\prime} ; x} .
$$

Received May, 1999. Revised August, 1999. Accepted September, 1999.

* Faculty of Economics, Okayama University, 3-1-1 Naka, Tsushima, Okayama 700-8530, Japan.

** Faculty of Economics, The University of Tokyo, 7-3-1 Hongo, Bunkyo-ku, Tokyo 1130033, Japan. 
This rule is equivalent to the allocation according to the geodesic distance $d$ on $S^{n-1}: d(\boldsymbol{x}, \boldsymbol{y})=\cos ^{-1}(\langle\boldsymbol{x}, \boldsymbol{y}\rangle), \boldsymbol{x}=\left(x_{1}, \cdots, x_{n}\right)^{\prime}, \boldsymbol{y}=\left(y_{1}, \cdots, y_{n}\right)^{\prime} \in S^{n-1},\langle\boldsymbol{x}, \boldsymbol{y}\rangle=$ $\sum_{i=1}^{n} x_{i} y_{i}$. Specifically, (1.1) is equivalent to

$$
d\left(\boldsymbol{x}, \boldsymbol{\mu}_{i}\right)<d\left(\boldsymbol{x}, \boldsymbol{\mu}_{j}\right) \text {. }
$$

Thus, the discriminant boundary $B_{i j}$ between populations $i$ and $j$ is the set of points on $S^{n-1}$ equidistant from $\boldsymbol{\mu}_{i}$ and $\boldsymbol{\mu}_{j}$ in terms of $d$ :

$$
B_{i j}=\left\{\boldsymbol{x} \in S^{n-1}: d\left(\boldsymbol{x}, \boldsymbol{\mu}_{i}\right)=d\left(\boldsymbol{x}, \boldsymbol{\mu}_{j}\right)\right\} .
$$

Now, $S^{n-1}$ is divided into regions by $m(m-1) / 2$ such boundaries $B_{i j}, 1 \leq i<j \leq$ $m$, and each region is indexed by a ranking of $\{1,2, \cdots, m\}$ in which $i$ is ranked better than $j$ iff (1.2) holds with an arbitrary point $x$ in the region.

However, as in the case of discrimination in $R^{n}$, when $n$ is small compared with $m$, not all the $m$ ! rankings are generated. Here we ask the same question as in the case of $R^{n}$ : How many rankings are generated from the discrimination on $S^{n-1}$ ? Actually, our regions on $S^{n-1}$ correspond to the unbounded regions of the linear discriminant analysis in $R^{n}$ treated in our previous paper KT. Hence, as would be clear for the readers of KT, in the case of discrimination on $S^{n-1}$, we have that for each pair of mutually reverse rankings of $\{1,2, \cdots, m\}$, both of them or neither of them is generated. That is, a ranking is generated if and only if its reverse ranking is generated.

The organization of this paper is as follows. After explaining background concepts in Section 2, we give the main result of this paper in Section 3. In the final section, we state several results of independent interest.

\section{Background concepts and definitions}

In this section, we introduce background concepts from rankings and hyperplane arrangements. We first explain some concepts concerning rankings.

A ranking of $m$ items $\{1,2, \cdots, m\}$ can be expressed as an ordering of them. The ordering $\sigma=\left(i_{1}, i_{2}, \cdots, i_{m}\right)$ corresponds to the ranking in which item $i_{1}$ is ranked first, item $i_{2}$ is ranked second, and so on. For an ordering $\sigma=\left(i_{1}, \cdots, i_{m}\right)$, the reverse ordering is defined to be $-\sigma=\left(i_{m}, \cdots, i_{1}\right)$. A partial ordering $\pi$ corresponds to a partial ranking, in which ties are allowed. Here we follow the convention that the order of items in braces is irrelevant; in parentheses it is relevant. So the partial ordering $\pi=(\{2,4\}, 3,1)$, for example, corresponds to the partial ranking in which items 2 and 4 are ranked first, item 3 is ranked third, and item 1 is ranked last. We attach the adjective "full" to ranking (ordering) when we want to emphasize the distinction from a partial ranking (ordering).

Next, we give a brief introduction to the theory of hyperplane arrangements. For a full treatment of the theory, the reader is referred to Chapters 1 and 2 of Orlik and Terao (1992).

A hyperplane arrangement $\boldsymbol{A}$ is defined to be a finite set of hyperplanes in $V=R^{n}$. The problem of counting chambers, i.e., regions, of a hyperplane 
arrangement becomes very hard when degeneracy is allowed. Zaslavsky (1975) gave a formula for the number of regions in an arbitrary arrangement $\boldsymbol{A}$ of hyperplanes. He introduced the method of deletion and restriction to obtain a recursion formula for chamber counting problems. By proving that the Poincaré polynomial evaluated at $1, \pi(A, 1)$, satisfies the same recursion, he obtained a beautiful result: The number of regions is equal to $\pi(A, 1)$.

We list some basic definitions from the theory of hyperplane arrangements. A hyperplane arrangement $\boldsymbol{A}$ is said to be centered if $\cap_{H \in A} H \neq \emptyset$. In particular, it is said to be central if each hyperplane contains the origin. The intersection poset (partially ordered set) $L=L(A)$ is defined to be the set of nonempty intersections of elements of $\boldsymbol{A}$ endowed with the partial order defined by

$$
X \leq Y \Longleftrightarrow Y \subseteq X \text {. }
$$

The rank function on $L$ is defined by the codimension: $r(X)=n-\operatorname{dim}(X)$, where $\operatorname{dim}(X)$ is the dimension of $X$. Maximal elements of $L(\boldsymbol{A})$ have the same rank, and the rank $r(\boldsymbol{A})$ of $\boldsymbol{A}$ is defined to be the rank of a maximal element of $L(\boldsymbol{A})$. Let $L_{p}=L_{p}(\boldsymbol{A})=\{X \in L(\boldsymbol{A}) \mid r(X)=p\}$. The Hasse diagram of $L$ is, by definition, the diagram which has vertices labeled by the elements of $L$ and arranged on levels $L_{p}$ for $p \geq 0$, and whose edges connect $X \in L_{p}$ with $Y \in L_{p+1}$ iff $X<Y$. Define the Möbius function $\nu$ as follows:

$$
\left\{\begin{array}{l}
\nu(V)=1 \\
\nu(X)=-\Sigma_{V \leq Y<X} \nu(Y) \text { if } V<X .
\end{array}\right.
$$

The Poincare polynomial of $\boldsymbol{A}$ is defined by

$$
\pi(A, t)=\sum_{X \in L} \nu(X)(-t)^{r(X)},
$$

where $t$ is an indeterminate. A chamber is defined to be a connected component of $\left\{x \in R^{n}: x \notin \cup_{H \in A} H\right\}$. However, in this paper we use the term "region" instead of "chamber."

Now we specialize to the arrangement of discriminant hyperplanes in the pairwise linear discriminant analysis among $m$ populations $N_{n}\left(\boldsymbol{\mu}_{i}, I\right), i=1, \cdots$, $m$, in $R^{n}$. Although in this paper we are concerned with discrimination on $S^{n-1}$, the discriminant boundaries on $S^{n-1}$ can be identified with the discriminant hyperplanes in $R^{n}$ as shown in Section 3.2.

Denote by $H_{i j}, 1 \leq j<i \leq m$, the discriminant hyperplane between populations $i$ and $j$ :

$$
H_{i j}=\left\{x \in R^{n}:\left(\boldsymbol{\mu}_{i}-\boldsymbol{\mu}_{j}\right)^{\prime}\left(x-\frac{1}{2}\left(\boldsymbol{\mu}_{i}+\boldsymbol{\mu}_{j}\right)\right)=0\right\},
$$

and consider the arrangement of discriminant hyperplanes

$$
\boldsymbol{A}=\left\{H_{i j}: 1 \leq j<i \leq m\right\} .
$$

Each element of $L=L(\boldsymbol{A})$ can be indexed by a partition $J$ of $m$ indices into blocks, and $X \leq Y$ for $X, Y \in L$ corresponds to the fact that the corresponding 
partition of $X$ is a refinement of that of $Y$, so that each block of the latter is a union of some blocks of the former. Specifically, to $X \in L$ corresponds the partition of $\{1,2, \cdots, m\}$ into equivalence classes under the equivalence relation $\sim_{x}$ defined by

$$
i \sim_{X} j \Longleftrightarrow X \subseteq H_{i j},
$$

where we agree that $H_{i i}=V$ and $H_{j i}=H_{i j}$ for $i>j$. Note that $i \sim_{x} j$ means any $\boldsymbol{x} \in X$ is equidistant from $\boldsymbol{\mu}_{i}$ and $\boldsymbol{\mu}_{j}$. The element of $L$ indexed by a partition $J$ is denoted by $X_{J}$.

The $m$ populations are ranked according to the Euclidean distances to $\boldsymbol{\mu}_{i}$. The population $i$ with the nearest $\boldsymbol{\mu}_{i}$ is ranked first; $j$ with the farthest $\boldsymbol{\mu}_{j}$ last. Thus, each region is indexed by a full ordering. Note that a region is open in $R^{n}$. Elements of $L$ of rank $n$, if they exist, are called terminal nodes, and can be indexed by partial orderings.

Regions fall into two types: bounded regions and unbounded ones. This distinction played. an important role in the characterization of non-generated rankings in $\mathrm{KT}$ (Section 3 of $\mathrm{KT}$ ). Also, there is a connection between unbounded regions and ideal vectors. See Section 4.3 of KT. In general, ideal vector model is defined as follows: Objects or items $1,2, \cdots, m$ are represented as points $\boldsymbol{x}_{1}, \boldsymbol{x}_{2}, \cdots, \boldsymbol{x}_{m}$ in $R^{n}$ for some $n$, and the "ideal vector" $\boldsymbol{d}$ is supposed to exist. Then the $m$ objects are ranked according to the projections onto this direction. Specifically, $i$ is ranked better than $j$ iff $\left.\left\langle\boldsymbol{d}, \boldsymbol{x}_{i}\right\rangle\right\rangle\left\langle\boldsymbol{d}, \boldsymbol{x}_{j}\right\rangle$.

\section{The main result}

In this section, we give a formula for the number of regions in pairwise discriminant analysis of $m$ populations $M_{n}\left(\boldsymbol{\mu}_{i}, \kappa\right), i=1, \cdots, m, \kappa>0$, on $S^{n-1}$ under rule (1.1).

We begin by defining non-degeneracy of our discriminant analysis on $S^{n-1}$.

\subsection{Definition of non-degeneracy}

We say that our discriminant analysis is non-degenerate if the following condition holds:

(A) The points $\mu_{1}, \cdots, \mu_{m} \in S^{n-1}$ regarded as points in $R^{n}$ are in general position (in the sense of Remark 3.1 below).

REMARK 3.1. When $m \leq n+1$, we say that $m$ points $x_{1}, \cdots, x_{m} \in R^{n}$ are in general position iff for any set of scalars $a_{1}, \cdots, a_{m}$ satisfying $\sum_{i} a_{i}=0$, equation $\sum_{i} a_{i} \boldsymbol{x}_{i}=\mathbf{0}$ implies $a_{i}=0$ for all $i=1, \cdots, m$.

When $m>n+1$, we say that $x_{1}, \cdots, x_{m} \in R^{n}$ are in general position iff any $n$ +1 of them are in general position.

Now we move on to the problem of finding the number of regions. We solve this problem by identifying our discrimination on $S^{n-1}$ with linear discriminant analysis in $R^{n}$. 


\subsection{Identification with discrimination in $\boldsymbol{R}^{n}$}

Regarding $\boldsymbol{\mu}_{1}, \cdots, \boldsymbol{\mu}_{m} \in S^{n-1}$ as points in $R^{n}$, we see that (1.2) is equivalent to the following rule by means of hyperplanes in $R^{n}$ :

$$
\left(\boldsymbol{\mu}_{i}-\boldsymbol{\mu}_{j}\right)^{\prime} \boldsymbol{x}>0 .
$$

Denote the hyperplanes in (3.1) by $H_{i j}$ :

$$
H_{i j}=\left\{\boldsymbol{x} \in R^{n_{0}}:\left(\boldsymbol{\mu}_{i}-\boldsymbol{\mu}_{j}\right)^{\prime} \boldsymbol{x}=0\right\}, 1 \leq j<i \leq m .
$$

Since each $H_{i j}, 1 \leq j<i \leq m$, contains the origin, we see that the arrangement $\boldsymbol{A}$ of these hyperplanes in $R^{n}$ :

$$
\boldsymbol{A}=\left\{H_{i j}: 1 \leq j<i \leq m\right\}
$$

is central. Accordingly, the closure of each region of $\boldsymbol{A}$ is a polyhedral cone. Hence, each of the rankings corresponding to these regions in $R^{n}$ appears as a region on $S^{n-1}$ when $S^{n-1}$ is embedded in $R^{n}$ (see Lemma 3.2 of KT). Note that (1.2) is equivalent to

$$
\left\|\boldsymbol{x}-\boldsymbol{\mu}_{i}\right\|<\left\|\boldsymbol{x}-\boldsymbol{\mu}_{j}\right\|
$$

Conversely, it is clear that each ranking on $S^{n-1}$ appears as a region in $R^{n}$. Therefore, we see that the sets of generated rankings agree for the discriminations in $R^{n}$ and on $S^{n-1}$.

The above argument implies that, instead of our original discrimination on $S^{n-1}$, we may consider the discrimination in $R^{n}$ by hyperplanes $H_{i j}$ in (3.2). Thus we can resort to the theory of the arrangements of hyperplanes as in our previous paper $\mathrm{KT}$, but the linear discriminant analysis in $R^{n}$ of the present paper differs from that of $\mathrm{KT}$ : The linear discrimination of the present paper is "degenerate" in the sense of KT, since the arrangement $\boldsymbol{A}$ in (3.3) is central. In summary, our non-degenerate discriminant analysis on $S^{n-1}$ can be identified with a degenerate one in $R^{n}$-degenerate in the sense that the arrangement of discriminant hyperplanes is central.

In accordance with this degeneracy, Lemma 2.2 of $\mathrm{KT}$ should be modified as follows. Recall that for the non-degenerate linear discriminant analysis of $m$ populations in $R^{n}$, the intersection poset $L\left(\boldsymbol{A}^{\prime}\right)$ of the arrangement $\boldsymbol{A}^{\prime}$ of discriminant hyperplanes is isomorphic to the poset $\mathcal{J}_{m-n}$ of partitions of $\{1,2$, $\cdots, m$ \} into not less than $m-n$ blocks (KT, Lemma 2.2). For the degenerate case (i.e., non-degenerate spherical discrimination), on the other hand, we need a slight modification in the following way.

LEMMA 3.1. For the non-degenerate discriminant analysis of $m$ populations on $S^{n-1}, n \leq m-1$, we have that $L=L(A)$ is isomorphic to the poset $\mathcal{J}_{m-n}$ : $=\mathcal{Z}_{m-n+1} \cup\{\{1,2, \cdots, m\}\}$ of partitions of $\{1,2, \cdots, m\}$ into one or $k \geq m-n+1$ blocks. Namely, $J \in \mathcal{J}_{m-n} \mapsto X_{J} \in L$ is bijective and $J_{1}$ is a refinement of $J_{2}$ iff $X_{J_{1}} \leq X_{J_{2}}$. Furthermore, 


$$
r\left(X_{J}\right)= \begin{cases}m-k, & J \in \mathcal{J}_{m-n+1} \text { with } k \geq m-n+1 \text { blocks, } \\ n, & J=\{\{1,2, \cdots, m\}\} .\end{cases}
$$

ProOF. First, note that in general, the set of points $x \in R^{n}$ equidistant from $x_{1}, x_{2}, \cdots, x_{l} \in S^{n-1}$ :

$$
\left\{x \in R^{n}:\left\|x-x_{1}\right\|^{2}=\cdots=\left\|x-x_{i}\right\|^{2}\right\}
$$

is equal to the set of points $x$ satisfying

$$
\left(\begin{array}{c}
x_{2}^{\prime}-x_{1}^{\prime} \\
x_{3}^{\prime}-x_{2}^{\prime} \\
\vdots \\
x_{l}^{\prime}-x_{l-1}^{\prime}
\end{array}\right) x=0
$$

where $\mathbf{0}=(0, \cdots, 0)^{\prime} \in R^{l-1}$.

Now, without loss of generality, consider the partition of $\{1, \cdots, m\}$ into $k$ blocks

$$
\left\{\left\{1, \cdots, l_{1}\right\},\left\{l_{1}+1, \cdots, l_{1}+l_{2}\right\}, \cdots,\left\{m-l_{k}+1, \cdots, m\right\}\right\}
$$

with $l_{1} \geq l_{2} \geq \cdots \geq l_{\vec{k}}>l_{\tilde{k}+1}=\cdots=l_{k}=1$, and the corresponding system of linear equations $\boldsymbol{M x}=\mathbf{0}$, where

$$
M=\left(\begin{array}{c}
\boldsymbol{\mu}_{2}^{\prime}-\boldsymbol{\mu}_{1}^{\prime} \\
\vdots \\
\boldsymbol{\mu}_{l_{1}}^{\prime}-\boldsymbol{\mu}_{l_{1-1}}^{\prime} \\
\boldsymbol{\mu}_{l_{1}+2}^{\prime}-\boldsymbol{\mu}_{l_{1+1}}^{\prime} \\
\vdots \\
\boldsymbol{\mu}_{l_{1}+l_{2}}^{\prime}-\boldsymbol{\mu}_{l_{1}+l_{2}-1}^{\prime} \\
\vdots \\
\boldsymbol{\mu}_{l_{1}+\cdots+l_{\tilde{k}-1}+2}^{\prime}-\boldsymbol{\mu}_{l_{1}+\cdots+l_{\tilde{k}-1}+1}^{\prime} \\
\vdots \\
\boldsymbol{\mu}_{l_{1}+\cdots+l_{\tilde{k}}}^{\prime}-\boldsymbol{\mu}_{h_{1}+\cdots+l_{k}-1}^{\prime}
\end{array}\right),
$$

$\boldsymbol{x} \in R^{n}$, and $\boldsymbol{0} \in R^{m-k}$. Here, $M$ is of size $(m-k) \times n$, and by the assumption of non-degeneracy, its rank is $\min \{m-k, n\}$. Hence, the space $\boldsymbol{S}$ of solutions $\boldsymbol{x}$ to $M \boldsymbol{x}=\mathbf{0}$ satisfies

$$
\begin{cases}\operatorname{dim} \boldsymbol{S}=n-(m-k) & \text { when } k>m-n, \\ \boldsymbol{S}=\{\mathbf{0}\} & \text { when } k \leq m-n .\end{cases}
$$

From this observation, the assertions of the lemma can be seen quite easily.

\subsection{The number of regions}

We are now in a position to state the main result of this paper.

THEOREM 3.1. The number of rankings generated by the non-degenerate discriminant analysis of $m$ populations on $S^{n-1}$ is given by 


$$
\begin{cases}2\left(c_{n-1}+c_{n-3}+\cdots+c_{0}\right), & n: \text { odd }, \\ 2\left(c_{n-1}+c_{n-3}+\cdots+c_{1}\right), & n: \text { even },\end{cases}
$$

where $c_{0}=1$ and

$$
c_{k}=\sum_{1 \leq l_{1}<l_{2}<\cdots<l_{k} \leq m-1} l_{1} l_{2} \cdots l_{k}, k \geq 1,
$$

in which $l_{1}, l_{2}, \cdots, l_{k}$ are natural numbers.

Note that $c_{k}$ are the unsigned Stirling numbers of the first kind.

PROOF. As was stated earlier, we may, instead, count the number of regions for the arrangement $\boldsymbol{A}$ of hyperplanes $H_{i j}$ in (3.2).

First, we treat the case $n \leq m-1$.

Our intersection poset $L(\boldsymbol{A})$ is isomorphic to $\tilde{\mathcal{J}}_{m-n}$, while in the nondegenerate case of $\mathrm{KT}, L\left(\boldsymbol{A}^{\prime}\right)$ is isomorphic to $\mathcal{J}_{m-n}$ (See Lemma 3.1 and the paragraph just above that). By comparing $\tilde{\mathcal{J}}_{m-n}$ and $\mathcal{Z}_{m-n}$, we see the Hasse diagram of our $L(\boldsymbol{A})$ is the same as that of $L\left(\boldsymbol{A}^{\prime}\right)$ except that the vertices of rank $n$ degenerate to a single vertex with index $\{\{1, \cdots, m\}\}$, connected by edges with all vertices of rank $n-1$.

Since the Poincaré polynomial of $\boldsymbol{A}^{\prime}$ was

$$
\pi\left(\boldsymbol{A}^{\prime}, t\right)=\sum_{l=0}^{n}(-1)^{l} c_{l}(-t)^{l}
$$

(KT, Proof of Theorem 2.1), the sum of the values of the Möbius function at rank $l$ was $(-1)^{l} c_{l}$. Thus the Möbius function in the present case takes the same values at ranks less than $n$ and $-\sum_{l \leq n-1}(-1)^{l} c_{l}$ at rank $n$.

Therefore, the Poincaré polynomial of the present case is

$$
\pi(\boldsymbol{A}, t)=\sum_{l \leq n-1} c_{l} t^{l}+\left(-\sum_{l \leq n-1}(-1)^{l} c_{l}\right)(-t)^{n}
$$

and the number of regions is

$$
\begin{aligned}
\pi(\boldsymbol{A}, 1) & =\sum_{l \leq n-1}\left(1+(-1)^{l+n+1}\right) c_{l} \\
& =2\left(c_{n-1}+c_{n-3}+c_{n-5}+\cdots\right) .
\end{aligned}
$$

The proof for the case $n \leq m-1$ is complete.

The formula is still valid for the case $n>m-1$ because of

$$
\begin{aligned}
2\left(c_{m-2}+c_{m-4}+\cdots\right) & =m !, \\
c_{m-1}+c_{m-3}+\cdots & =c_{m-2}+c_{m-4}+\cdots
\end{aligned}
$$

(KT, p.21) and $c_{i}=0$ for $i \geq m$.

REMARK 3.2. Perturb the $m$ points $\boldsymbol{\mu}_{1}, \cdots, \boldsymbol{\mu}_{m} \in S^{n-1}$ slightly in $R^{n}$ in such a way that 


$$
\left(\boldsymbol{\mu}_{1}^{\prime},\left\|\boldsymbol{\mu}_{1}\right\|^{2}\right), \cdots,\left(\boldsymbol{\mu}_{m}^{\prime},\left\|\boldsymbol{\mu}_{m}\right\|^{2}\right)
$$

are in general position in $R^{n+1}$ as well as $\boldsymbol{\mu}_{1}, \cdots, \boldsymbol{\mu}_{m}$ in $R^{n}$ (KT, p.12). Then, comparing the two arrangements before and after the perturbation, we see that the arrangement before perturbation is obtained by degenerating all the terminal nodes of the arrangement after perturbation to a single point. Accordingly, regions of the linear discrimination in $R^{n}$ of the present paper are expected to correspond to regions of unbounded regions of non-degenerate discriminant analysis in $R^{n}$ of KT. Indeed, the number of unbounded regions in $\mathrm{KT}$ is

$$
\left(c_{n}+c_{n-1}+\cdots+c_{0}\right)-\left(c_{n}-c_{n-1}+c_{n-2}-\cdots\right)=2\left(c_{n-1}+c_{n-3}+\cdots\right)
$$

(KT, Theorems 2.1 and 2.3), which agrees with the result in Theorem 3.1.

REMARK 3.3. As is seen from Theorem 3.1, the number of rankings generated by discriminant analysis on $S^{n-1}$ is always even. This can be understood by considering for each $x \in S^{n-1}$ its antipodal point $-x \in S^{n-1}$. That is, for each pair of mutually reverse rankings, both of them or neither of them is generated.

\section{Miscellaneous results}

Other results in $\mathrm{KT}$ for linear discrimination in $R^{n}$ remain to hold with minor modifications in our spherical discrimination.

First, the shortest path in terms of the usual metric on $S^{n-1}$ is also the shortest path in terms of a particular distance for orderings. Specifically, the numerator of Kendall's $\tau$ between two generated orderings-that is, the minimum number of pairwise adjacent transpositions needed to transform one ordering to the other-is equal to the number of discriminant boundaries $B_{i j}$ meeting the geodesic path on $S^{n-1}$ between points of the two corresponding regions. The proof is almost the same as that of Theorem 4.1.1 of KT.

Secondly, we can characterize the regions around a terminal node in the following way. First note that, in view of the identification with discrimination in $R^{n}$ in Section 3.2, a terminal node in our spherical discrimination-that is, a point on $S^{n-1}$ given as the intersection of some of the discriminant boundaries $B_{i j}-$ can be indexed by a partial ordering with $m-n+1$ blocks

$$
\pi=\left(\pi_{1}, \cdots, \pi_{m-n+1}\right) .
$$

Now, when $n \leq m$, around a terminal node $x \in S^{n-1}$ corresponding to partial ordering (4.1), there arise those, and only those, regions which are obtained by giving arbitrary orders to items in $\pi_{1}, \cdots, \pi_{m-n+1}$ independently. The proof is similar to that of Theorem 4.2.1 of KT.

Thirdly, the equivalence of our ranking rule and the rule by ideal vector model in $R^{n}$ is obvious from the equivalence of (1.2) and $\left.\left\langle x, \mu_{i}\right\rangle\right\rangle\left\langle x, \mu_{j}\right\rangle, x \in$ $S^{n-1}$. 


\section{Acknowledgment}

We thank Prof. Shinto Eguchi for valuable comments. We also thank the referees for useful suggestions which led to much improvement of the paper.

\section{REFERENCES}

[1] Kamiya, H. and Takemura, A. (1997). On rankings generated by pairwise linear discriminant analysis of $m$ populations. J. Multivariate Anal., 61, 1-28.

[2] Mardia, K. V., Kent, J. T., and Bibby, J. M. (1979) Multivariate Analysis. Academic Press, San Diego.

[ 3 ] Orlik, P. and Terao, H. (1992). Arrangements of Hyperplanes. Springer-Verlag, Berlin.

[ 4 ] Zaslavsky, T. (1975). Facing up to Arrangements: Face-Count Formulas for Partitions of Space by Hyperplanes. American Mathematical Society, Providence, RI. 